\title{
The role of peptide receptor radionuclide therapy in advanced/ metastatic thoracic neuroendocrine tumors
}

\author{
Lisa Bodei ${ }^{1}$, Jarosław B. Ćwikla ${ }^{2}$, Mark Kidd $^{3}$, Irvin M. Modlin ${ }^{4}$ \\ ${ }^{1}$ Molecular Imaging and Therapy Service, Memorial Sloan Kettering Cancer Center, New York, NY, USA; ${ }^{2}$ Faculty of Medical Sciences, University \\ of Warmia and Mazury, Olsztyn, Poland; ${ }^{3}$ Wren Laboratories, Branford, CT, USA; ${ }^{4}$ Department of Surgery, Yale University School of Medicine, \\ New Haven, CT, USA \\ Contributions: (I) Conception and design: All authors; (II) Administrative support: None; (III) Provision of study materials or patients: None; (IV) \\ Collection and assembly of data: All authors; (V) Data analysis and interpretation: All authors; (VI) Manuscript writing: All authors; (VII) Final \\ approval of manuscript: All authors. \\ Correspondence to: Lisa Bodei, MD, PhD. Molecular Imaging and Therapy Service, Department of Radiology, Memorial Sloan Kettering Cancer \\ Center, 1275 York Avenue, Box 77, New York, NY 10065, USA. Email: bodeil@mskcc.org.
}

\begin{abstract}
Bronchopulmonary (BP) neuroendocrine tumors (NETs) comprise a spectrum of tumors that develop from respiratory neuroendocrine cells and represent $\sim 20 \%$ of all lung neoplasia and $\sim 30 \%$ of all NETs. The only curative treatment is surgical resection. For well-differentiated forms (typical and atypical carcinoids), medical therapy ranges from bioactive agents (e.g., somatostatin analogs), to biotherapy (e.g., everolimus), standard chemotherapy and peptide receptor radionuclide therapy (PRRT). PRRT with radiolabeled somatostatin analogs is an innovative treatment for inoperable or metastasized, well/moderately differentiated, NET. Initially developed for gastroenteropancreatic tumors, it is also used in BP-NET because these tumors express the target receptor. Two decades of clinical trials with either ${ }^{90} \mathrm{Y}$-octreotide or ${ }^{177} \mathrm{Lu}$-octreotate, have demonstrated the efficacy of PRRT, as measured by tumor response, symptom relief and quality of life (QoL) improvement. PRRT with ${ }^{90} \mathrm{Y}$ - and ${ }^{177} \mathrm{Lu}$-peptides is generally well-tolerated and adverse events (kidney and bone marrow) are modest. The paper illustrates the history, technique and results of this treatment in the few dedicated studies and the many BP NET cases embedded within larger NET series. The limitations of the present body of information are addressed, and the future perspectives, in terms of prospective studies required to define the position of PRRT in the therapeutic algorithm of BP-NETs and the need for predictive molecular biomarkers to guide future studies, are discussed.
\end{abstract}

Keywords: Bronchopulmonary (BP); neuroendocrine tumor (NET); peptide receptor radionuclide therapy (PRRT); ${ }^{177} \mathrm{Lu}$-octreotate; ${ }^{90} \mathrm{Y}$-octreotide

Submitted Aug 15, 2017. Accepted for publication Sep 15, 2017.

doi: $10.21037 /$ jtd.2017.09.82

View this article at: http://dx.doi.org/10.21037/jtd.2017.09.82

\section{Introduction}

Neuroendocrine tumors (NETs) arise both in the gastroentero-pancreatic tract and in the bronchopulmonary (BP) system (Figure 1) (1). BP NETs comprise a spectrum of tumors that specifically arise from respiratory neuroendocrine cells. They represent $\sim 20 \%$ of all lung neoplasia and $\sim 30 \%$ of all NETs (2). BP-NETs may present with cough, hemoptysis, and obstructive pneumonia but this depends on their site, size, and growth pattern. They are frequently identified serendipitously on chest radiology. Less than $5 \%$ exhibit hormonally-related symptoms such as carcinoid syndrome, Cushing, acromegaly, or SIADH.

BP-NETs are divided into four subgroups: typical carcinoid tumor (TC), atypical carcinoid tumor (AC), largecell neuroendocrine carcinoma (LCNEC), and small-cell 


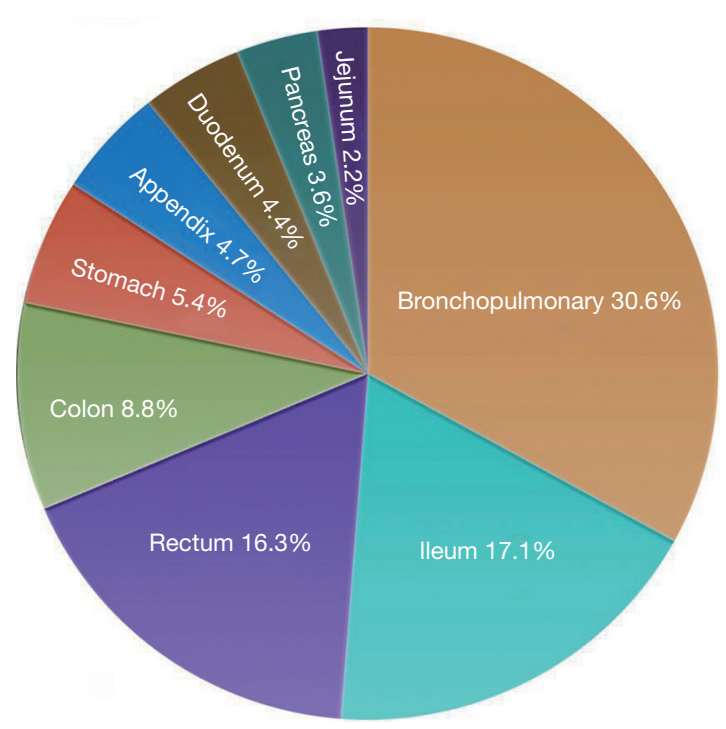

Figure 1 Incidence of different types of neuroendocrine tumors. The majority are located in the gastro-entero-pancreatic tract and the lung.

lung carcinoma (SCLC). Both SCLC and LCNEC progress rapidly, are aggressively metastatic, with a poor prognosis. TC generally behaves in a benign/indolent fashion, whereas AC exhibit indolent to highly aggressive behavior. SCLC is the most common variant, whereas LCNEC is the rarest. The only curative treatment is surgical resection. TC exhibits a fairly good prognosis (5-year survival, 90\%), although metastases may appear years after apparently radical resection of the primary. The 5 -year survival of AC is $50-80 \%$ consistent with a more aggressive phenotype. The highly malignant LCNEC and SCLC exhibit poorer prognoses, $30 \%$ and $<5 \%$, respectively $(2,3)$.

Given the widely different biological behavior between different types of BP-NETs, and the development of liver metastases, treatment is mostly focused on either the removal of the primary lesion or the management of metastatic disease. Ideally, removal of the primary tumor should be initially undertaken and thereafter, appropriate strategies developed for the management of residual disease. This is usually undertaken in a multidisciplinary fashion and is individualized according to the histological type e.g., TC or AC and burden, as well as symptomatology. For unresectable tumors, optimal selection of palliative treatment options (timing and modality) is of paramount importance to maintain or improve quality of life (QoL) and prolong overall survival (Figure 2).
The surgical options include resection of the primary (wedge resection or lobectomy, and in extreme cases pneumonectomy), hepatic resection of metastases, radiofrequency ablation, stereotactic body radiation therapy (SBRT) and even hepatic transplantation $(4,5)$. Interventional radiology techniques include embolization of hepatic metastases (with or without cytotoxic agents) or the use of radioactive microspheres. Medical therapy ranges from the use of bioactive agents such as somatostatin analogs or interferon to standard chemotherapy. More recently, a variety of novel molecular targeted agents including the mTOR inhibitor Everolimus have been utilized with marginal efficacy (3). Among chemotherapeutic agents, temozolomide has been tested in several non-randomized trials, alone or in combination with targeted therapies in patients with well- and intermediately differentiated BPNETs with some activity (6). Of particular interest has been the development of targeted radiotherapy using a variety of different isotopes including Indium, Yttrium and Lutetium (7). This novel therapeutic strategy, delivered by intravenous infusion has been designated peptide receptor radionuclide therapy (PRRT).

\section{PRRT background}

PRRT with radiolabeled somatostatin analogs is an innovative treatment for inoperable or metastasized, well/ moderately differentiated, NETs (7). Initially developed for gastroenteropancreatic tumors, it is also used in BPNET because these tumors express the target receptor (8). Radiolabeled somatostatin analogs represent, to date, the prototype and the most successful paradigm of theranostics, namely of molecules exploiting the same receptor binding and internalization mechanism for imaging and therapy. This reflects the development of the synthetic somatostatin analog peptides, which are octreotide and the variety of radio-labelled variants. The therapeutic efficacy is related to a high affinity for somatostatin receptors subtype 2 (sst2) and moderate affinity for subtype 5 (sst5). A high proportion $(67 \%)$ of bronchial NETs over-expresses somatostatin receptors, especially the sst2 subtype (9).

Binding of an isotopically labeled radiopeptide to somatostatin receptors expressed on the cell membrane of NET results in internalization and delivery of the radioactivity directly into the intracellular space of the tumor cell (Figure 3). This retention of intracellular radiation is associated with DNA damage through betaemission and subsequent apoptosis through an inability of 


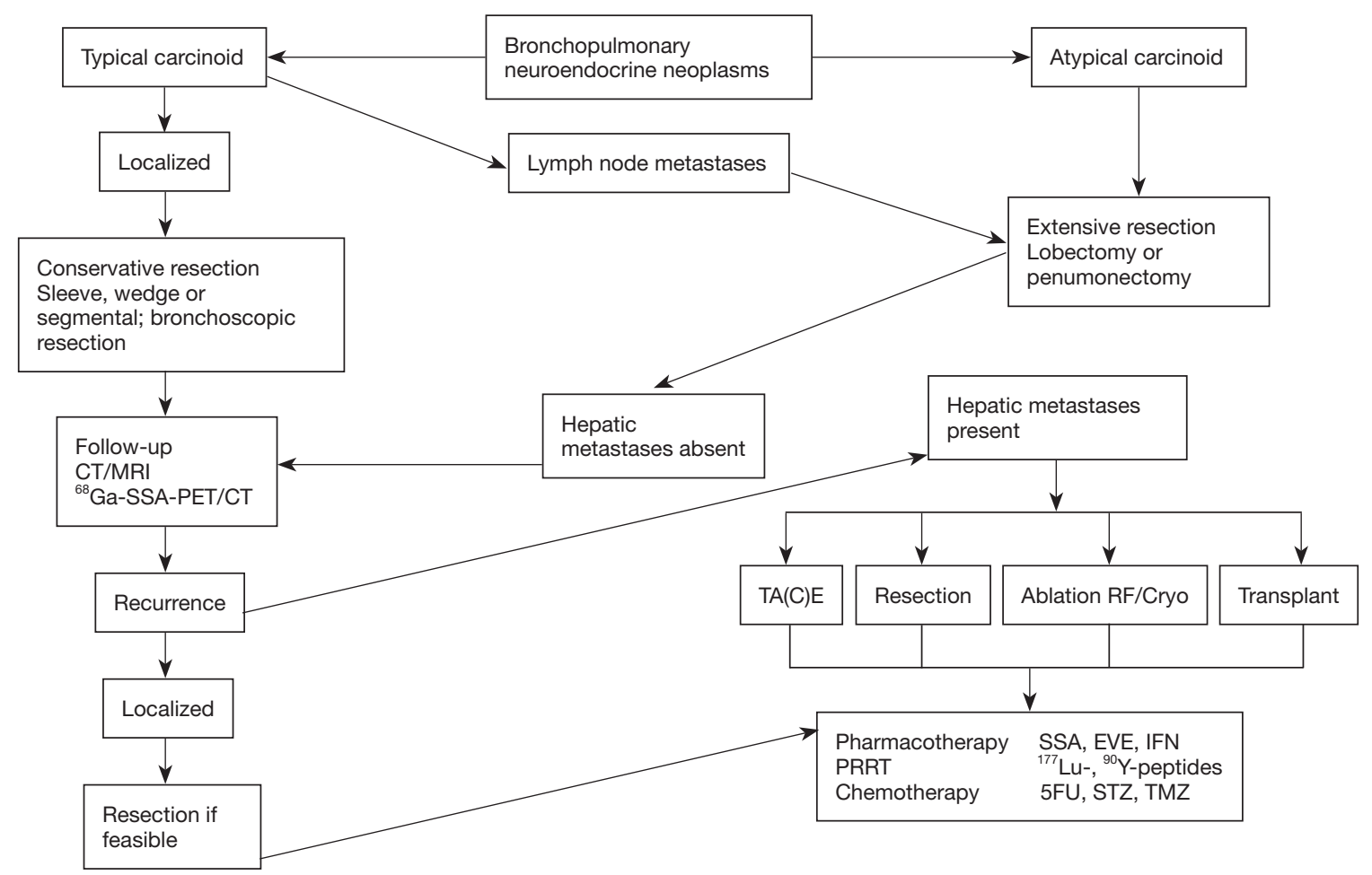

Figure 2 Treatment options in bronchopulmonary NETs (typical and atypical carcinoids). In metastatic or unresectable disease locoregional strategies as well as systemic treatments, such as PRRT, are performed. PRRT, peptide receptor radionuclide therapy; NET, neuroendocrine tumor; STZ, streptozotocin; TMZ, temozolomide; SSA, somatostatin analog; EVE, everolimus; IFN, interferon.

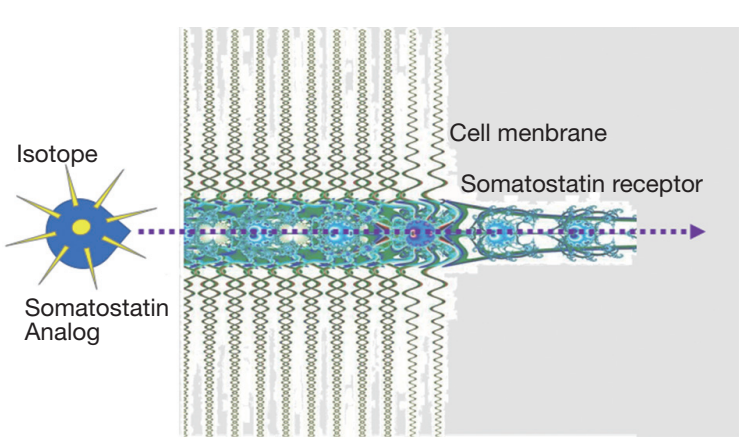

Figure 3 Mechanism of effectiveness of PRRT. After the somatostatin (SS) analog linked to the isotope binds to the membrane somatostatin receptor, the radiopeptide/somatostatin receptor complex is internalized. Thus, radioactivity is transported into the intracellular receptor recycling compartment of the NET cell where it exerts its action in proximity to the nucleus. PRRT, peptide receptor radionuclide therapy. the cell to correct the damage. Other internal structures including mitochondria may be destroyed by radiation.

Clinically, PRRT comprises the systemic administration of a suitably radiolabeled synthetic somatostatin analog, fractionated into sequential cycles (usually 4-5) every 6 to 9 weeks, until the intended total amount of radioactivity has been delivered. The precise amount administered is dependent on the limitations imposed by renal irradiation and, to a lesser extent, bone marrow sensitivity.

PRRT was introduced into clinical practice in 1994. This was the logical theranostic application following the development of diagnostic techniques for localization of NET using the radiolabeled somatostatin analog $\left({ }^{111} \mathrm{In}-\right.$ DTPA0-D-Phe1)-octreotide or ${ }^{111}$ In-pentetreotide (10). Using the same principle, but with an increased isotope activity (high-dose ${ }^{111}$ In-pentetreotide), a theranostic was developed. Therapeutic efficacy was linked to the activity of the Auger and conversion electrons emitted by ${ }^{111}$ Indium (low) and consequently disease remission was rare (11). This led to the use of isotopes with higher energy and longer range, such as the pure beta emitter ${ }^{90} \mathrm{Yttrium}$. The particles 


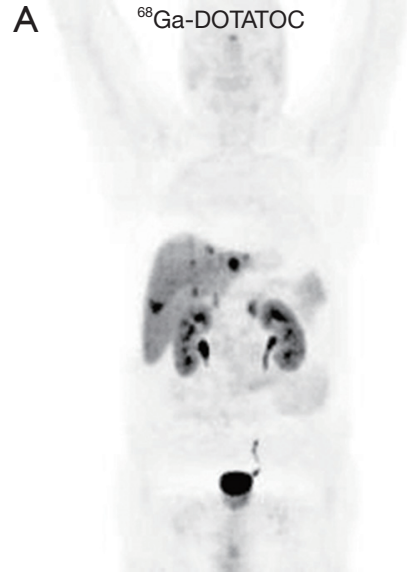

Maximum intensity projection image

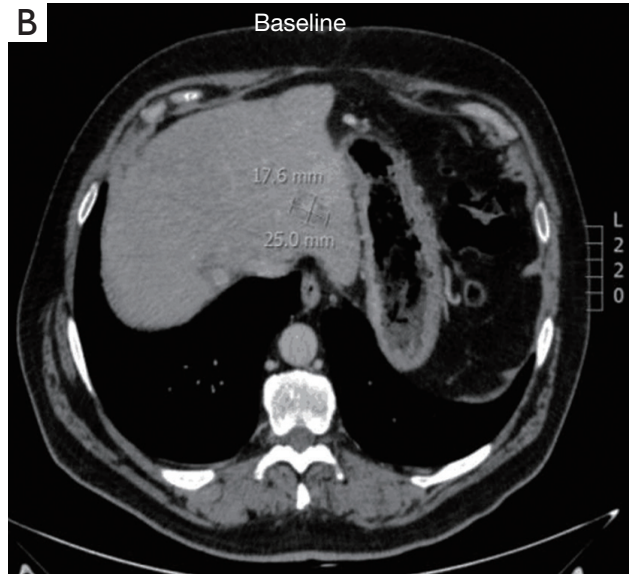

Liver CT, axial image

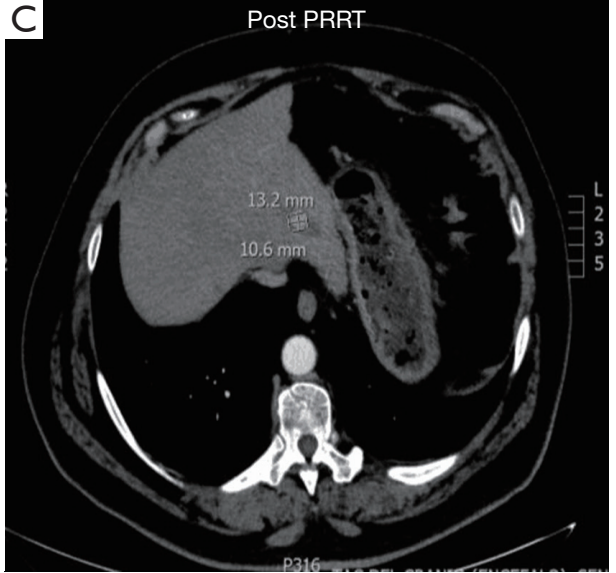

Liver CT, axial image

Figure 4 Patient affected by liver metastases from an atypical carcinoid, previously treated with surgery and SSA. The 68Ga-DOTATOC PET/CT (A, maximum intensity projection image) shows elevated SSR expression in the tumor lesions (B, baseline CT). The patient is treated with ${ }^{177} \mathrm{Lu}$-DOTATATE (cumulative activity $27.8 \mathrm{GBq}$ ), obtaining objective response (C, follow-up CT scan). PRRT, peptide receptor radionuclide therapy; SSA, somatostatin analog; SSR, somatostatin receptor.

emitted by ${ }^{90} \mathrm{Y}$ (maximum energy $2.27 \mathrm{MeV}$, penetration range $R_{\beta \max } 11 \mathrm{~mm}$, half-life $T_{1 / 2} 64 \mathrm{hrs}$ ) allowed for a direct killing of somatostatin receptor-positive cells and a crossfire effect that targeted nearby receptor-negative tumor cells. Novel octreotide analogs were also developed to increase efficacy. For ${ }^{90} \mathrm{Y}$, a new analog, $\mathrm{Tyr}^{3}$-octreotide, with a similar pattern of affinity for somatostatin receptors, was developed at the University of Basel. This analog was characterized by high hydrophobicity, ease of labeling with ${ }^{111} \mathrm{In}$ and ${ }^{90} \mathrm{Y}$, and tight binding to the bifunctional chelator DOTA, which securely encloses the radioisotope (1,4,7,10-tetraazacyclododecane-N,N',N",N'"-tetra-acetic acid) $(12,13)$.

$\left({ }^{90} \mathrm{Y}\right.$-DOTA $\left.{ }^{0}, \mathrm{Tyr}^{3}\right)$-octreotide or ${ }^{90} \mathrm{Y}$-DOTATOC or ${ }^{90} \mathrm{Y}$-octreotide was initially used in the treatment of metastatic NETs in 1996 (7). The excellent symptomatic and objective response following several cycles of ${ }^{90} \mathrm{Y}$-octreotide therapy encouraged further studies to examine the potential of PRRT in NET disease (14). As a consequence, ${ }^{90} \mathrm{Y}$-octreotide became the most utilized radiopeptide in the first decade of PRRT experience (15-19).

Since 2000, a more effective analog, octreotate $\left(\mathrm{Tyr}^{3}\right.$, Thr ${ }^{8}$-octreotide) with 6- to 9-fold higher affinity for sst2, was developed and clinically utilized. The chelated analog (DOTA $)^{0}-\mathrm{Tyr}^{3}$-octreotate or DOTATATE can be labeled with the $\beta-\gamma$ emitter Lutetium-177 $\left(\mathrm{E}_{\beta \max } 0.49 \mathrm{MeV}, \mathrm{R}_{\beta \max }\right.$ $2 \mathrm{~mm}, \mathrm{~T}_{1 / 2} 6.7$ days) and has been investigated in several clinical phase I and II studies $(15,20,21) .{ }^{177} \mathrm{Lu}$-octreotate has subsequently become one of the most frequently utilized radiopeptides for PRRT. This has been particularly evident in recent studies given its efficacy, tolerability and manageability $(22,23) .{ }^{177} \mathrm{Lu}$-octreotate has recently being evaluated in a randomized phase III registration trial in small bowel NET.

\section{PRRT clinical protocol}

Candidates for PRRT are individuals with tumors exhibiting a significant over-expression of somatostatin receptors. Inclusion criteria also include the functionality of somatostatin receptors, namely their ability to internalize the receptor-analog complex and retain the radioactivity inside the cell.

Individuals are selected based on the results of scintigraphy with ${ }^{111}$ In-pentetreotide (or, more recently, receptor PET with ${ }^{68}$ Gallium-labeled octreotide). Appropriate candidates have image results indicative of adequate uptake (at least equal to the uptake of normal liver) as evidence of targetable somatostatin receptors. This is necessary to ensure and calculate an appropriate high tumor dose with low exposure to normal tissues that express physiological levels of somatostatin receptors (24).

Scintigraphic or PET tomoscintigraphic evaluation is the most accurate noninvasive method to identify and confirm the over-expression of functioning receptors (25). Optimally, as opposed to immunohistochemistry determination, the use of in vivo functional scintigraphic 
or PET methods facilitates the simultaneous evaluation of the receptor density and the internalization capacity in real time in all lesions, which is useful for therapeutic selection of patients (Figure 4).

When evaluating images to determine PRRT selection, it is important to exclude false positives. False positives include uptake in the gall bladder (inflammation), accessory spleens, recent surgical scars (inflammatory infiltrate), previous radiotherapy and any other cause of granulomatous-lymphoid infiltrate that can mimic the presence of NET tissue. The signal typically represents accumulation of inflammatory cells which express somatostatin receptors.

False negatives should also be considered. These are mainly represented by small, sub-centimeter lesions, below the resolution limit of the instrument (although this limitation is partially overcome by receptor PET/CT). In addition, certain tumors, such as the majority of highly malignant and high grade (Ki67 >55\%) NETs do not express adequate numbers of detectable somatostatin receptors.

\section{PRRT technique}

PRRT consists of the systemic administration of a radiolabeled somatostatin analog. The radiopeptide is intravenously administered slowly over 20-30 minutes in approximately $100 \mathrm{~mL}$ of saline. The cumulative activity, fractionated in multiple cycles, is calculated from imaging results to efficiently irradiate the tumor, without surpassing the tolerance dose threshold of the kidneys and bone marrow, in an individualized fashion. The timing of fractionation, every 6 to 9 weeks, is based upon calculations regarding recovery times from possible acute hematological toxicity (7).

To diminish renal dose of irradiation, patients are premedicated with an intravenous infusion of positively charged amino acids (lysine or arginine) in the amount of $\sim 25 \mathrm{~g}$ per day. This infusion is initiated $30-60$ minutes prior to the administration of the isotope and is maintained until 2-3 hours following cessation of the isotope infusion. The infusion both hydrates the patients and reduces the renal radioactivity dose by competitively inhibiting the proximal tubular reabsorption of the radiopeptide. In some circumstances, mild adverse events are experienced. These include gastro-intestinal symptoms such as a slight nausea, and occasionally emesis. These may be related to the amino acid co-administration, but are easily controlled with appropriate medication.

\section{PRRT efficacy}

Two decades of clinical application with either ${ }^{90}$ Y-octreotide or ${ }^{177} \mathrm{Lu}$-octreotate, has demonstrated PRRT is an effective clinical therapy as measured by tumor response, symptom relief and QoL improvement (Table 1).

The recent NETTER-1 phase III randomized trial of ${ }^{177} \mathrm{Lu}-$ DOTATATE $v s$. high-dose Octreotide LAR in patients with inoperable, progressive, midgut carcinoid tumors identified that ${ }^{177} \mathrm{Lu}$-octreotate significantly improves PFS (PFS not reached vs. 8.4 months; hazard ratio 0.21 , with a $79 \%$ reduction of the risk of progression) (33). The overall number of deaths was also significantly lower in the PRRT group (14 vs. 26).

\section{PRRT and BP NET}

Three recent studies have examined the utility of PRRT in BP-NETs. In the first, a phase II study that was performed in individuals with "poor responding" tumors including bronchial and gastric NECs. Patients were treated with standard 22.2-29.6 GBq activities. The results for BP-NETs were: 5 partial responses, 1 minor response and 2 stabilizations in nine patients. In this series, PRRT was as effective in bronchial NETs as had been noted in GEP-NETs (34).

In a recent, larger study ( $\mathrm{n}=34$ bronchial NETs), the disease control rate was $80 \%$, with $6 \%$ achieving a complete response, $27 \%$ a partial response and $47 \%$ disease stabilization. The overall median progression-free survival (mPFS) was 20.1 months (95\% CI: 11.8-26.8 months) for the group. Interestingly, the mPFS was shorter in patients with positive TTF-1 (thyroid transcription factor 1: $\mathrm{n}=18$; 7.2 vs. 26.3 months). This reflects a more aggressive pattern. In addition, a positive FDG PET/CT ( $\mathrm{n}=16)$, which is reflective of more metabolically active and aggressive disease was also associated with a shorter mPFS (15.3 vs. 26.4 months) (35).

A retrospective review of 114 advanced bronchial NETs treated with ${ }^{90}$ Yttrium-, a combination of ${ }^{90} \mathrm{Y}$ and ${ }^{177}$ Lutetium-, or ${ }^{177} \mathrm{Lu}$-based PRRT protocols at IEO Milan, identified the median overall survival to be 58.8 months, with an mPFS of 28 months. Patients treated with ${ }^{177} \mathrm{Lu}-$ DOTATATE, alone or in combination with ${ }^{90} \mathrm{Y}$-PRRT $(\mathrm{n}=48$ and 21 , respectively) exhibited the longest 5 -year overall survival $(61.4 \%$ for both series, vs. $31.6 \%$ for ${ }^{90}$ Y-PRRT). No factor clinical factor(s) was identified that could accurately measure efficacy of therapy in BP-NETs or predict those patients who would benefit from PRRT (36). 
Table 1 Clinical results of PRRT with either ${ }^{90}$ Y-octreotide or ${ }^{177}$ Lu-octreotate in GEP NETs

\begin{tabular}{|c|c|c|c|c|c|c|c|c|}
\hline Agent & Schedule & Patients & $\mathrm{CR}$ & PR & DCR & $\begin{array}{l}\text { Progression } \\
\text { at baseline }\end{array}$ & $\begin{array}{l}\text { Response } \\
\text { criteria }\end{array}$ & $\begin{array}{l}\text { Outcome (median } \\
\text { PFS or TTP) }\end{array}$ \\
\hline \multirow[t]{4}{*}{${ }^{90} \mathrm{Y}$-octreotide } & $\begin{array}{l}7.4 \mathrm{GBq} / \mathrm{sqm} \text { in } \\
4 \text { cycles }(26)\end{array}$ & 36 GEP & $4 \%$ & $20 \%$ & $92 \%$ & $100 \%$ & WHO & NA \\
\hline & $\begin{array}{l}2.96-5.55 \mathrm{GBq} / \\
\text { cycle } \times 2(16)\end{array}$ & 21 GEP & $0 \%$ & $28 \%$ & $71 \%$ & NA & WHO & TTP 10 m \\
\hline & $\begin{array}{l}\text { 4.4 GBq/cycle } \\
\times 3(18)\end{array}$ & $90 \mathrm{SI}$ & $0 \%$ & $4 \%$ & $74.4 \%$ & $100 \%$ & SWOG & PFS 16 m \\
\hline & $\begin{array}{l}1-10 \text { cycles } \\
\text { (median } 2) \text {, various } \\
\text { activity (19) }\end{array}$ & $\begin{array}{l}821 \\
\text { GEP }\end{array}$ & $0.2 \%$ & $38 \%$ & NA & NA & RECIST & NA \\
\hline \multirow{4}{*}{${ }^{177}$ Lu-octreotate } & $\begin{array}{l}3.7-29.2 \mathrm{GBq} \text { in } \\
4-6 \text { cycles of } \\
3.7-7.4 \mathrm{GBq}(21)\end{array}$ & 39 GEP & $3 \%$ & $31 \%$ & $88 \%$ & $76 \%$ & RECIST & TTP 36 months \\
\hline & $\begin{array}{l}\text { Mean } 25.5 \mathrm{GBq} \text { in } \\
5 \text { cycles, normal } \\
\text { subjects; mean } \\
17.8 \mathrm{GBq} \text { in risk } \\
\text { patients }(28)\end{array}$ & $52 \mathrm{P}$ & $8 \%$ & $21 \%$ & $81 \%$ & $88 \%$ & SWOG & $\begin{array}{l}\text { PFS } 20 \text { months in } \\
\text { reduced dosage, } \\
\text { not reached in full } \\
\text { dosage }\end{array}$ \\
\hline & $\begin{array}{l}32 \text { GBq in } \\
4 \text { cycles (29) }\end{array}$ & $68 \mathrm{P}$ & $0 \%$ & $60.3 \%$ & $85.3 \%$ & $67.6 \%$ & SWOG & PFS 34 months \\
\hline & $\begin{array}{l}27.8-29.6 \mathrm{GBq} \\
\text { in } 3-4 \text { cycles vs. } \\
\text { octreotide LAR } \\
60 \mathrm{mg} / \mathrm{month}(32)\end{array}$ & $201 \mathrm{SI}$ & $\begin{array}{l}19 \%(\mathrm{Lu}) \\
\text { (LAR) CR }\end{array}$ & $\begin{array}{l}\text { vs. } 3 \% \\
+\mathrm{PR}\end{array}$ & $\begin{array}{l}20 \%(\mathrm{Lu}) \\
\text { vs. } 58 \% \\
(\mathrm{LAR})\end{array}$ & $100 \%$ & RECIST & $\begin{array}{l}\text { PFS not reached } \\
\text { (Lu) vs. } 8.4 \text { months } \\
\text { (LAR) }\end{array}$ \\
\hline
\end{tabular}

PRRT, peptide receptor radionuclide therapy; GEP, gastroenteropancreatic; NET, neuroendocrine tumor; CR, complete response; PR, partial response; DCR, disease control rate; SI, small intestine; Lu, lutetium; LAR, long-acting release; PFS, progression-free survival; TTP, time to progression; NA, not assessed;

\section{Efficacy of individual peptides}

\section{${ }^{90}$ Y-octreotide}

Information regarding ${ }^{90} \mathrm{Y}$ efficacy in BPNETs is difficult to evaluate at this time as the original studies were heterogeneous in composition. Nevertheless, some observations can be made.
In a study carried out at Basel University, 39 patients with NETs, mostly of GEP origin, were treated with 4 cycles of ${ }^{90}$ Y-octreotide, with a cumulative activity of $7.4 \mathrm{GBq}$. Three patients with progressive bronchial tumors were included. All demonstrated disease stabilization after PRRT (37). The group as a whole had 2 complete remissions, 7 partial responses and 27 disease stabilization. 
The results of two phase I-II studies and a retrospective evaluation in 141 patients were published by the Milan group in 2004 (38). NETs, principally of the gastro-enteropancreatic $(\mathrm{n}=58 ; 41.1 \%)$ and bronchial tract $(\mathrm{n}=12 ; 8.5 \%)$ were treated with a cumulative activity of 7.4-26.4 GBq of ${ }^{90} \mathrm{Y}$-octreotide, divided into $2-16$ cycles, administered 4-6 weeks apart. In the 11 patients with bronchial tumors, $10(91 \%)$ were in progression at enrolment, and were treated with standard courses of PRRT, with cumulative activities ranging from 8 to $22.5 \mathrm{GBq}$. After completion of the treatment, 1 patient had a partial remission and 8 showed stabilization of disease (SWOG criteria). In an earlier escalation study published by the same group in patients with somatostatin positive tumors (mainly in progression), 3 patients with bronchial NETs were included, with resulting stability and partial remission in 2 (17).

More recently, the Basel group published the results of an open-label phase II trial in 1,109 patients treated with ${ }^{90} \mathrm{Y}$-octreotide, divided into multiple cycles of $3.7 \mathrm{GBq} / \mathrm{m}^{2}$ each. In this series, 84 bronchial NET were treated. The rate of objective response was $28.6 \%$. This was similar to small bowel NET (26.8\%) but less than for pancreatic NET (47\%). The best predictor of survival, however, was the tumor uptake at baseline (19).

\section{${ }^{90} \mathrm{Y}-+{ }^{177} \mathrm{Lu}$-peptide combinations}

Protocols combining ${ }^{177} \mathrm{Lu}$ - and ${ }^{90} \mathrm{Y}$-peptides have been recently considered in order to take advantage of the different physical properties of both two radionuclides. Theoretically, the combination of the two radioisotopes should allow simultaneous treatment of large lesions (based on the higher energy and penetration range of the particles emitted by ${ }^{90} \mathrm{Y}$ ) as well as on small lesions (based on the lower energy and penetration range of ${ }^{177} \mathrm{Lu}$ ). The results of PRRT performed in a Danish cohort of 69 patients treated in Basel with different combinations of Y- and/or Lu-peptides were recently published. Six patients with bronchial NETs were included; 1 exhibited a partial remission and 3 exhibited stabilization (39).

\section{${ }^{177}$ Lu-octreotate}

${ }^{177} \mathrm{Lu}$-DOTATATE or ${ }^{177} \mathrm{Lu}$-octreotate is currently the most commonly used radiopeptide for PRRT. It has a higher affinity for the somatostatin sst2 receptor, it is easier to use, it results in a lower dosimetric burden on the kidney because of lower radiation, and it allows radiologists to obtain scintigraphic images and undertake dosimetric studies at the same time, owing to the gamma photon coemission of ${ }^{177} \mathrm{Lu}$.

A cohort of 51 patients with unresectable/metastatic NETs, 5 of which of BP origin, were treated in a phase I-II study in Milan aimed at defining toxicity and efficacy of ${ }^{177} \mathrm{Lu}$-octreotate. Patients were divided into two groups, receiving escalating activities, from 3.7-5.18 GBq and from 5.18-7.4 GBq, with cumulative activities up to $29 \mathrm{GBq}$, based on dosimetry. Partial and complete responses were observed in 15 patients (32.6\%) (21).

A salvage protocol with ${ }^{177} \mathrm{Lu}$-octreotate was published by the Rotterdam group. Patients in progression were enrolled after an initial response to PRRT with ${ }^{177} \mathrm{Lu}$ octreotate, administered using standard cumulative activities (22.2-29.6 GBq). In this series, 32 patients with bronchial or GEP-NETs received 2 additional cycles of ${ }^{177} \mathrm{Lu}$-octreotate, with a cumulative activity of $15 \mathrm{GBq}$. Nevertheless, this "salvage therapy" was well tolerated by the majority of patients and should be considered as a valuable option for subjects who exhibit progression on PRRT (40)

\section{PRRT safety profile}

Accumulated clinical experience and evidence accumulated over the past two decades and have demonstrated that PRRT with ${ }^{90} \mathrm{Y}$ - and ${ }^{177} \mathrm{Lu}$-peptides is generally welltolerated and that adverse events are modest. Acute side effects are usually mild (nausea and rarely, emesis) and are correlated to the (type of) co-administration of amino acids used to reduce renal exposure to radiation. Other adverse modest events (commonly, fatigue) and the exacerbation of an endocrine syndrome or hormonal crisis ( $1 \%$, mainly occurring in the treatment of functional tumors) represent the cytotoxic effects of the radiopeptide. Chronic and permanent effects in the kidneys and the bone marrow are generally mild if the necessary precautions (renal protection with amino acids; dosage fractionation and attention to specific risk factors e.g., hypertension or previous nephroor myelo-toxic chemotherapy regimens), are undertaken $(24,41)$ (Table 2).

Usage of appropriate dosimetry improves the delivery of elevated absorbed doses to the tumor, with relative sparing of healthy organs (kidneys, bone marrow) (45) (Table 3).

\section{Renal}

Renal irradiation occurs due to the reabsorption of the 
Table 2 Long-term toxicity after PRRT with either ${ }^{90}$ Y-octreotide or ${ }^{177} \mathrm{Lu}$-octreotate in GEP-NETs

\begin{tabular}{|c|c|c|c|c|c|c|}
\hline Agent & Patients & Follow-up (months) & Renal toxicity & MDS & Acute leukemia & Reference \\
\hline \multirow{3}{*}{${ }^{90}$ Y-octreotide } & 39 & 6 & $3 \%$ Grade 2 & 0 & 0 & (26) \\
\hline & 58 & 18 & $3 \%$ Grade 4 & 1 & 0 & (27) \\
\hline & 1,109 & 23 & $9.2 \%$ Grade $3 / 4$ & 1 & 1 & (19) \\
\hline \multirow[t]{4}{*}{${ }^{177}$ Lu-octreotate } & 504 & 19 & $0.4 \%$ Grade 4 & 3 & 0 & (20) \\
\hline & 51 & 29 & $24 \%$ Grade 1 & 0 & 0 & (21) \\
\hline & 74 & 21 & $1.3 \%$ Grade $3 / 4$ & 3 & 0 & $(43,44)$ \\
\hline & 290 & 30 & $0 \%$ & $6(2.06 \%)$ & $2(0.69 \%)$ & $(42)$ \\
\hline
\end{tabular}

PRRT, peptide receptor radionuclide therapy; GEP-NET, gastroenteropancreatic neuroendocrine tumor; MDS, myelodysplastic syndrome.

Table 3 Mean absorbed doses for ${ }^{90} \mathrm{Y}$-octreotide and ${ }^{177} \mathrm{Lu}$-octreotate

\begin{tabular}{lccccc}
\hline \multirow{2}{*}{ Organ } & \multicolumn{2}{c}{${ }^{90}{ }^{90}$-octreotide } & & \multicolumn{2}{c}{${ }^{177}$ Lu-octreotate } \\
\cline { 2 - 3 } \cline { 5 - 6 } Kidney* & Mean (Gy/GBq) & 13 GBq course (Gy) & & Mean (Gy/GBq) & 29 GBq course (Gy) \\
Bone marrow & $1.1-5.1$ & $15-66$ & $0.3-1.7$ & $0.3-2.3$ \\
Tumor & $0.02-0.2$ & $0.3-2.6$ & $0.01-0.08$ & $0.6-56$ & $17-1,624$ \\
\hline
\end{tabular}

Data derived from published studies (46) (with examples of standard full courses of therapy, using either 13 or 29 GBq). In general, absorbed doses to normal organs, e.g., kidney or bone marrow, are variable on an individual basis. Specific tumor absorbed doses are dependent on the level of radioactivity concentration in individual lesions, and increase with radioactivity accumulation in the tumor. Tumor doses themselves are also highly variable based upon factors intrinsic to the tumor itself, particularly the density of somatostatin receptors on the tumor cell membranes, the dimension of the lesions and the distribution of radioactivity within the lesions. *, renal doses are calculated based upon the use of renal protection with amino acid solutions.

radio peptides in the proximal convoluted tubules, with a subsequent accumulation in the renal interstitium, where the radioactivity exerts its action inducing vasculitis and fibrosis (47). Renal toxicity is significantly decreased when positively charged amino acids, such as lysine and arginine, are co-infused with the radioligand. These competitively inhibit proximal tubular radiopeptide reabsorption by saturating the apical membrane megalin, resulting in a $9-53 \%$ reduction in renal radioactive dosage (48). Overall, a mild loss of renal function does occur over time, with a median decline in creatinine clearance of $7.3 \%$ per year for ${ }^{90}$ Y-octreotide and 3.8\% per year for ${ }^{177} \mathrm{Lu}$-octreotate (49). Severe, end-stage renal damage, however, remains rare with ${ }^{177} \mathrm{Lu}$-octreotate and only sporadic cases are reported in literature (15). Earlier concerns of high numbers of renal problems reported were mostly associated with the use of ${ }^{90}$ Y-peptide PRRT, and reflected administration of very high activities in the absence of amino acid induced renal protection or renal protection with low/nihil lysine and arginine amounts (50).

Overall PRRT with ${ }^{90} \mathrm{Y}$-peptides is more frequently associated with a reduction of renal function, presumably reflecting the specific physical characteristics of the ${ }^{90} \mathrm{Y}$ radionuclide, namely the much larger particle penetration into the kidney. A long-term evaluation of renal toxicity in a group $(\mathrm{n}=28)$ undergoing PRRT with dosimetric analysis showed that, of the 23 treated with ${ }^{90}$ Y-octreotide, a lower dose threshold for toxicity was observed in patients with risk factors (mainly hypertension and diabetes) (51).

In a retrospective series $(n=1,109)$ treated with 
${ }^{90} \mathrm{Y}$-octreotide, 103 subjects (9.2\%) experienced grade 4 to 5 permanent renal toxicity (19). Multivariate regression revealed that the initial kidney uptake was predictive of severe renal toxicity. However, it seems likely that this relatively high incidence was related to the highadministered activities per cycle $\left(3.7 \mathrm{GBq} / \mathrm{m}^{2}\right.$ body surface, namely activities of about $6.4 \mathrm{GBq}$ per cycle in a standard male). Furthermore, individuals with preexisting impairment of renal function were not excluded from PRRT and infusion of protective amino acids was not routinely employed in the earlier phases of the study (50).

Recently, the results of an analysis of long-term tolerability of PRRT $(\mathrm{n}=807)$ treated with ${ }^{90} \mathrm{Y}$-octreotide, ${ }^{177} \mathrm{Lu}$-octreotate or the combination (Lu: $34.4 \%$, Y: 44.4\%, $\mathrm{Lu}+\mathrm{Y}: 19.5 \%)$ provided additional information regarding PRRT toxicity. This analysis illuminated the lack of a clearly definable role for previous clinical parameters (such as diabetes, hypertension and chemotherapy) considered responsible. Nephrotoxicity, either transient or persistent, occurred in $279(34.6 \%)$; this was severe in $1.5 \% .{ }^{90}$ Y-peptides or the combination of ${ }^{90} \mathrm{Y} /{ }^{177} \mathrm{Lu}$-peptides showed greater G3/4 nephrotoxicity (2.8\% and $1.3 \%$, respectively) than ${ }^{177} \mathrm{Lu}$-peptides alone ( $\left.0 \%\right)$. However, less than $30 \%$ of risk for toxicity could be modeled by clinical parameters. Hypertension and anemia were noted to be the most relevant known risk factors. It seems probable that a unique individual susceptibility to toxicity, possibly of genetic origin, might play a role (42).

\section{Hematological}

From a hematological perspective, PRRT is generally well-tolerated. Subacute toxicity with severe WHO grade 3 or 4 toxicity occurs in $<13 \%$ after ${ }^{90}$ Y-octreotide and in $\sim 10 \%$ after ${ }^{177} \mathrm{Lu}$-octreotate. Nevertheless, sporadic cases of myelodysplastic syndrome (MDS) or even overt acute leukemia have been reported, in the order of $2 \%$ (41). Although predicted absorbed doses are lower than the conventional threshold for toxicity, both acute and permanent bone marrow damage remains a cause for concern, particularly in the event of repeated radionuclide administrations or heavy pretreatment with chemotherapy (52).

An analysis of 203 patients treated with 4 intended cycles of $8 \mathrm{GBq}$ each at 3-month intervals, had an incidence of MDS of $1.4 \%$. Myelo-suppression was almost invariably reversible and the cumulative administered activity and initial cytopenia were the most important risk factors for myelotoxicity (43).
The analysis of the 807 group identified that MDS occurred in $2.35 \%$ (42). The "classical" risk factors, such as chemotherapy were only associated with predicting risk of toxicity in $30 \%$. Clinical features, such as platelet toxicity grade and the increasing duration of PRRT, were, partially responsible. This led to speculation that unidentified individual genetic susceptibilities, may be related to developing radiation-associated disease (42).

More recent studies have demonstrated that ${ }^{177} \mathrm{Lu}-$ octreotate can be safely utilized even in florid bone metastases, with extreme bone replacement and the potentially higher exposure of bone marrow (53). Significant G3/G4 reversible hematological toxicity occurred in 35\% $(n=4)$ of 11 patients. Toxicity either resolved spontaneously ( 1 case) or was controlled by support therapy (3 cases). A return to baseline values was obtained in 23 months after completion of PRRT (53).

Finally, the issue of a potential increased toxicity of Everolimus, a bioactive drug frequently used in BP NETs, when administered after the completion of PRRT, has been examined in two studies $(54,55)$. This is important because everolimus has well described adverse effects, and the question is whether the combination therapy would yield a cumulative increase in toxicity. In a prospective study 24 subjects pretreated with PRRT had no significant adjunctive toxicity when treated with Everolimus, compared to the non-PRRT treated individuals from the literature (e.g., RADIANT-3 study). Major events were hyperglycemia (20.8\%), fatigue (8.3\%), thrombocytopenia (8.3\%) and elevated ALT (8.3\%) (54). These results differ to a retrospective multicentric study which indicated higher severe toxicity in patients previously treated with systemic chemotherapy and PRRT. This issue is incompletely resolved, due to the lack of robust prospective data, but remains of considerable clinical significance given the increasingly frequent use of sequential multidisciplinary strategies employing both treatments (55).

\section{Future advances}

A requirement to define the efficacy of PRRT is the need to more scientifically assess the parameters of response to the treatment. Specific molecular features including the identification of tumor radiation sensitivity markers, identification of lung-specific markers to quantify proliferation and apoptosis and easily accessibly molecular indices e.g., circulating tumor biomarkers 


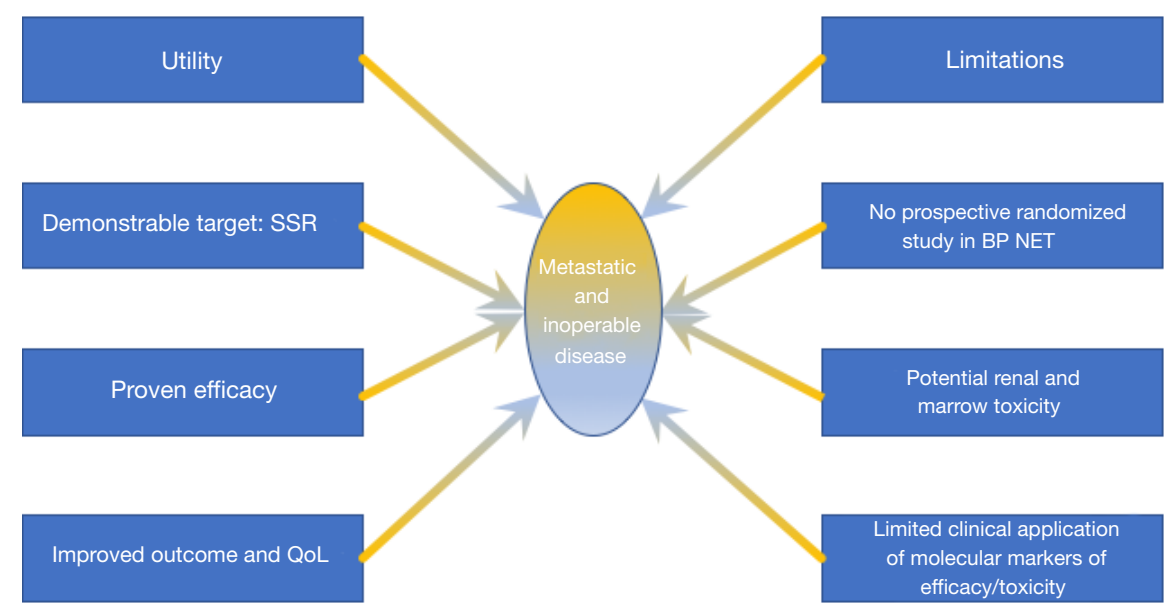

Figure 5 Utility and limitations of PRRT in the treatment of BP-NETs. PRRT, peptide receptor radionuclide therapy; BP, bronchopulmonary; NET, neuroendocrine tumor; SSR, somatostatin receptor.

that define response need delineation. Such information would augment and perhaps supplant the current tools such as image-based evidence of disease extension, quantification of basal isotope uptake at receptor imaging and the pathological and morphological assessments of the lesion type.

Some steps have already been undertaken. Pretreatment functional analysis of tumors utilizing metabolomic parameters conveys information in regard to the likelihood of radiation sensitivity (56). Recent studies have noted that FDG is a crucial parameter in predicting the duration of response to PRRT including in BP-NETs. Individuals with positive FDG exhibit a significantly shorter PFS, clear evidence that tumor glucose utilization represents a significant parameter in predicting therapeutic efficacy (57).

In a reality where prediction of outcome (efficacy, tolerability) becomes a major issue, in view of the multidisciplinary sequencing of long and expensive therapies, and where both conventional (morphologic/ functional) imaging and current biomarkers have demonstrated their limitations (Figure 5). As adjunct, molecular tools, such as transcript analysis of specific circulating NET mRNA signature have begun to show promising results. A circulating multianalyte 51-gene NET signature demonstrated significant advantage in early detection of residual disease of surgery-treated patient or in the assessment of somatostatin analog response $(58,59)$. Furthermore, this tool can accurately define and predict response to PRRT including in BPNET (60).

\section{Conclusions}

PRRT has become a well-accepted effective therapeutic modality for inoperable or metastatic GEP and now has an established role in the treatment of BP-NET. It is overall well-tolerated with the majority of recipients experiencing only moderate toxicity if the necessary precautions are undertaken. The two most commonly used radiopeptides, ${ }^{90}$ Y-octreotide and ${ }^{177} \mathrm{Lu}$-octreotate, produce significant objective response rates, with positive impact on PFS and OS. In addition, both biochemical and symptomatic responses are commonly observed. Sophisticated molecular tools need to be incorporated into the management strategy to more effectively define therapeutic efficacy and early identification of adverse events. The strategy of randomized controlled trials is a key issue to standardize the treatment and establish the position of PRRT in the therapeutic algorithm of BP-NETs.

\section{Acknowledgements}

None.

\section{Footnote}

Conflicts of Interest: The authors have no conflicts of interest to declare.

\section{References}

1. Modlin IM, Oberg K, Chung DC, et al. 
Gastroenteropancreatic neuroendocrine tumours. Lancet Oncol 2008;9:61-72.

2. Gustafsson BI, Kidd M, Chan A, et al. Bronchopulmonary neuroendocrine tumors. Cancer 2008;113:5-21.

3. Caplin ME, Baudin E, Ferolla P, et al. Pulmonary neuroendocrine (carcinoid) tumors: European Neuroendocrine Tumor Society expert consensus and recommendations for best practice for typical and atypical pulmonary carcinoids. Ann Oncol 2015;26:1604-20.

4. Doherty G. Surgical treatment of neuroendocrine tumors (including carcinoid). Curr Opin Endocrinol Diabetes Obes 2013;20:32-6.

5. Pericleous M, Karpathakis A, Toumpanakis C, et al. Welldifferentiated bronchial neuroendocrine tumours: Clinical management and outcomes in 105 patients. Clin Respir J 2016. [Epub ahead of print].

6. Crona J, Fanola I, Lindholm DP, et al. Effect of temozolomide in patients with metastatic bronchial carcinoids. Neuroendocrinology 2013;98:151-5.

7. Bodei L, Mueller-Brand J, Baum RP, et al. The joint IAEA, EANM, and SNMMI practical guidance on peptide receptor radionuclide therapy (PRRNT) in neuroendocrine tumours. Eur J Nucl Med Mol Imaging 2013;40:800-16.

8. Reubi JC, Waser B. Concomitant expression of several peptide receptors in neuroendocrine tumours: molecular basis for in vivo multireceptor tumour targeting. Eur J Nucl Med Mol Imaging 2003;30:781-93.

9. Reubi JC, Waser B. Triple-peptide receptor targeting in vitro allows detection of all tested gut and bronchial NETs. J Nucl Med 2015;56:613-5.

10. Krenning EP, Kooij PP, Bakker WH, et al. Radiotherapy with a radiolabeled somatostatin analogue, [111In-DTPAD-Phe1]-octreotide. A case history. Ann N Y Acad Sci 1994;733:496-506.

11. Valkema R, De Jong M, Bakker WH, et al. Phase I study of peptide receptor radionuclide therapy with [In-DTPA] octreotide: the Rotterdam experience. Semin Nucl Med 2002;32:110-22.

12. Heppeler A, Froidevaux S, Eberle AN, et al. Receptor targeting for tumor localisation and therapy with radiopeptides. Curr Med Chem 2000;7:971-94.

13. de Jong M, Bakker WH, Krenning EP, et al. Yttrium-90 and indium-111 labelling, receptor binding and biodistribution of [DOTA0,d-Phe1,Tyr3] octreotide, a promising somatostatin analogue for radionuclide therapy. Eur J Nucl Med 1997;24:368-71.

14. Otte A, Mueller-Brand J, Dellas S, et al. Yttrium-90- labelled somatostatin-analogue for cancer treatment. Lancet 1998;351:417-8.

15. Kwekkeboom DJ, Mueller-Brand J, Paganelli G, et al. Overview of results of peptide receptor radionuclide therapy with 3 radiolabeled somatostatin analogs. J Nucl Med 2005;46 Suppl 1:62s-6s.

16. Bodei L, Cremonesi M, Zoboli S, et al. Receptor-mediated radionuclide therapy with 90Y-DOTATOC in association with amino acid infusion: a phase I study. Eur J Nucl Med Mol Imaging 2003;30:207-16.

17. Bodei L, Handkiewicz-Junak D, Grana C, et al. Receptor radionuclide therapy with $90 \mathrm{Y}$-DOTATOC in patients with medullary thyroid carcinomas. Cancer Biother Radiopharm 2004;19:65-71.

18. Bushnell DL Jr, O'Dorisio TM, O'Dorisio MS, et al. 90Y-edotreotide for metastatic carcinoid refractory to octreotide. J Clin Oncol 2010;28:1652-9.

19. Imhof A, Brunner P, Marincek N, et al. Response, survival, and long-term toxicity after therapy with the radiolabeled somatostatin analogue [90Y-DOTA]-TOC in metastasized neuroendocrine cancers. J Clin Oncol 2011;29:2416-23.

20. Kwekkeboom DJ, de Herder WW, Kam BL, et al. Treatment with the radiolabeled somatostatin analog [177 Lu-DOTA 0,Tyr3] octreotate: toxicity, efficacy, and survival. J Clin Oncol 2008;26:2124-30.

21. Bodei L, Cremonesi M, Grana CM, et al. Peptide receptor radionuclide therapy with (1)(7)(7)Lu-DOTATATE: the IEO phase I-II study. Eur J Nucl Med Mol Imaging 2011;38:2125-35.

22. Bodei L, Kwekkeboom DJ, Kidd M, et al. Radiolabeled Somatostatin Analogue Therapy Of Gastroenteropancreatic Cancer. Semin Nucl Med 2016;46:225-38.

23. Strosberg J, El-Haddad G, Wolin E, et al. Phase 3 Trial of 177Lu-Dotatate for Midgut Neuroendocrine Tumors. N Engl J Med 2017;376:125-35.

24. Bodei L, Ferone D, Grana MC, et al. Peptide receptor therapies in neuroendocrine tumors. J Endocrinol Invest 2009;32:360-9.

25. Bodei L, Sundin A, Kidd M, et al. The Status of Neuroendocrine Tumor Imaging: From Darkness to Light? Neuroendocrinology 2015;101:1-17.

26. Waldherr C, Pless M, Maecke HR, et al. Tumor response and clinical benefit in neuroendocrine tumors after 7.4 GBq (90)Y-DOTATOC. J Nucl Med 2002;43:610-6.

27. Valkema R, Pauwels S, Kvols LK, et al. Survival and response after peptide receptor radionuclide therapy with [90Y-DOTA0,Tyr3] octreotide in patients with advanced 
gastroenteropancreatic neuroendocrine tumors. Semin Nucl Med 2006;36:147-56.

28. Sansovini M, Severi S, Ambrosetti A, et al. Treatment with the radiolabelled somatostatin analog Lu-DOTATATE for advanced pancreatic neuroendocrine tumors. Neuroendocrinology 2013;97:347-54.

29. Ezziddin S, Khalaf F, Vanezi M, et al. Outcome of peptide receptor radionuclide therapy with $177 \mathrm{Lu}$-octreotate in advanced grade 1/2 pancreatic neuroendocrine tumours. Eur J Nucl Med Mol Imaging 2014;41:925-33.

30. Paganelli G, Sansovini M, Ambrosetti A, et al. 177LuDota-octreotate radionuclide therapy of advanced gastrointestinal neuroendocrine tumors: results from a phase II study. Eur J Nucl Med Mol Imaging 2014;41:1845-51.

31. Sabet A, Dautzenberg K, Haslerud T, et al. Specific efficacy of peptide receptor radionuclide therapy with (177)Lu-octreotate in advanced neuroendocrine tumours of the small intestine. Eur J Nucl Med Mol Imaging 2015;42:1238-46.

32. AAA Announces Positive Results From Phase 3 Study NETTER-1 Evaluating Lutathera in Patients with Advanced Midgut Neuroendocrine Tumors. Available online: http://www.adacap.com/wp-content/ uploads/2015/09/2015-09-27-Press-Release-NETTER-1results-ENG-FINAL-FINAL1.pdf

33. Strosberg J, Wolin EM, Chasen B, et al. 177-Lu-Dotatate significantly improves progression-free survival in patients with midgut neuroendocrine tumours: Results of the phase III NETTER-1 trial. Eur J Cancer 2015;51:S710.

34. van Essen M, Krenning EP, Bakker WH, et al. Peptide receptor radionuclide therapy with $177 \mathrm{Lu}$-octreotate in patients with foregut carcinoid tumours of bronchial, gastric and thymic origin. Eur J Nucl Med Mol Imaging 2007;34:1219-27.

35. Ianniello A, Sansovini M, Severi S, et al. Peptide receptor radionuclide therapy with (177)Lu-DOTATATE in advanced bronchial carcinoids: prognostic role of thyroid transcription factor 1 and (18)F-FDG PET. Eur J Nucl Med Mol Imaging 2016;43:1040-6.

36. Mariniello A, Bodei L, Tinelli C, et al. Long-term results of PRRT in advanced bronchopulmonary carcinoid. Eur J Nucl Med Mol Imaging 2016;43:441-52.

37. Waldherr C, Pless M, Maecke HR, et al. Tumor response and clinical benefit in neuroendocrine tumors after 7.4 GBq (90)Y-DOTATOC. J Nucl Med 2002;43:610-6.

38. Bodei L, Cremonesi M, Grana C, et al. Receptor radionuclide therapy with $90 \mathrm{Y}$-[DOTA]0-Tyr3-octreotide
(90Y-DOTATOC) in neuroendocrine tumours. Eur J Nucl Med Mol Imaging 2004;31:1038-46.

39. Pfeifer AK, Gregersen T, Grønbæk H, et al. Peptide Receptor Radionuclide Therapy with ${ }^{90} \mathrm{Y}$-DOTATOC and ${ }^{177}$ Lu-DOTATOC in Advanced Neuroendocrine Tumors: Results from a Danish Cohort Treated in Switzerland. Neuroendocrinology 2011;93:189-96.

40. van Essen M, Krenning EP, Kam BL, et al. Salvage therapy with (177)Lu-octreotate in patients with bronchial and gastroenteropancreatic neuroendocrine tumors. J Nucl Med 2010;51:383-90.

41. Kwekkeboom DJ, Krenning EP. Peptide Receptor Radionuclide Therapy in the Treatment of Neuroendocrine Tumors. Hematol Oncol Clin North Am 2016;30:179-91.

42. Bodei L, Kidd M, Paganelli G, et al. Long-term tolerability of PRRT in 807 patients with neuroendocrine tumours: the value and limitations of clinical factors. Eur J Nucl Med Mol Imaging 2015;42:5-19.

43. Sabet A, Ezziddin K, Pape UF, et al. Long-term hematotoxicity after peptide receptor radionuclide therapy with 177Lu-octreotate. J Nucl Med 2013;54:1857-61.

44. Sabet A, Ezziddin K, Pape UF, et al. Accurate assessment of long-term nephrotoxicity after peptide receptor radionuclide therapy with (177)Lu-octreotate. Eur J Nucl Med Mol Imaging 2014;41:505-10.

45. Sandstrom M, Garske-Roman U, Granberg D, et al. Individualized dosimetry of kidney and bone marrow in patients undergoing 177Lu-DOTA-octreotate treatment. J Nucl Med 2013;54:33-41.

46. Cremonesi M, Botta F, Di Dia A, et al. Dosimetry for treatment with radiolabelled somatostatin analogues. A review. Q J Nucl Med Mol Imaging 2010;54:37-51.

47. Vegt E, Melis M, Eek A, et al. Renal uptake of different radiolabelled peptides is mediated by megalin: SPECT and biodistribution studies in megalin-deficient mice. Eur J Nucl Med Mol Imaging 2011;38:623-32 .

48. Bernard BF, Krenning EP, Breeman WA, et al. D-lysine reduction of indium-111 octreotide and yttrium-90 octreotide renal uptake. J Nucl Med 1997;38:1929-33.

49. Valkema R, Pauwels SA, Kvols LK, et al. Long-term follow-up of renal function after peptide receptor radiation therapy with (90)Y-DOTA(0), Tyr(3)-octreotide and (177) Lu-DOTA(0), Tyr(3)-octreotate. J Nucl Med 2005;46 Suppl 1:83s-91s.

50. Cybulla M, Weiner SM, Otte A. End-stage renal disease after treatment with 90Y-DOTATOC. Eur J Nucl Med 2001;28:1552-4. 
51. Bodei L, Cremonesi M, Ferrari M, et al. Long-term evaluation of renal toxicity after peptide receptor radionuclide therapy with $90 \mathrm{Y}-\mathrm{DOTATOC}$ and $177 \mathrm{Lu}-$ DOTATATE: the role of associated risk factors. Eur J Nucl Med Mol Imaging 2008;35:1847-56.

52. Bodei L, Modlin IM, Luster M, et al. Myeloid neoplasms after chemotherapy and PRRT: myth and reality. Endocr Relat Cancer 2016;23:C1-7.

53. Sabet A, Khalaf F, Yong-Hing CJ, et al. Can peptide receptor radionuclide therapy be safely applied in florid bone metastases? A pilot analysis of late stage osseous involvement. Nuklearmedizin 2014;53:54-9.

54. Kamp K, Gumz B, Feelders RA, et al. Safety and efficacy of everolimus in gastrointestinal and pancreatic neuroendocrine tumors after (177)Lu-octreotate. Endocr Relat Cancer 2013;20:825-31.

55. Panzuto F, Rinzivillo M, Fazio N, et al. Real-world study of everolimus in advanced progressive neuroendocrine tumors. Oncologist 2015;20:570.

56. Bodei L, Kidd M, Modlin IM, et al. Measurement of circulating transcripts and gene cluster analysis predicts

Cite this article as: Bodei L, Ćwikla JB, Kidd M, Modlin IM. The role of peptide receptor radionuclide therapy in advanced/ metastatic thoracic neuroendocrine tumors. J Thorac Dis 2017;9(Suppl 15):S1511-S1523. doi: 10.21037/jtd.2017.09.82 and defines therapeutic efficacy of peptide receptor radionuclide therapy (PRRT) in neuroendocrine tumors. Eur J Nucl Med Mol Imaging 2016;43:839-51.

57. Severi S, Nanni O, Bodei L, et al. Role of 18FDG PET/ CT in patients treated with $177 \mathrm{Lu}$-DOTATATE for advanced differentiated neuroendocrine tumours. Eur J Nucl Med Mol Imaging 2013;40:881-8.

58. Modlin IM, Frilling A, Salem RR, et al. Blood measurement of neuroendocrine gene transcripts defines the effectiveness of surgical resection and ablation strategies. Surgery 2016;159:336-47.

59. Cwikla JB, Bodei L, Kolasinska-Cwikla A, et al. Circulating transcript analysis (NETest) in GEP-NETs treated with Somatostatin Analogs defines Therapy. J Clin Endocrinol Metab 2015:jc20152792.

60. Bodei L, Kidd M, Modlin IM, et al. Gene transcript analysis blood values correlate with Ga-DOTAsomatostatin analog (SSA) PET/CT imaging in neuroendocrine tumors and can define disease status. Eur J Nucl Med Mol Imaging 2016;43:839-51. 